\title{
Fatores materno-infantis associados ao desenvolvimento de bebês prematuros e a termo
}

\author{
Maternal-infant factors associated to the development of preterm and full-term babies \\ Factores materno-infantiles asociados al desarrollo de bebés prematuros y a término \\ Rafaela Almeida Schiavo ${ }^{1}$ \\ Universidade Paulista (UNIP)
}

Olga Maria Piazentin Rolim Rodrigues

Universidade Estadual Paulista (UNESP)

Janaina Senhorini dos Santos

Juliana Marinho Antonucci

Centro Especializado de Reabilitação SORRI-Bauru

Carolina Mormanno

Universidade Estadual Paulista (UNESP)

Verônica Aparecida Pereira

Universidade Federal da Grande Dourados (UFGD)

\begin{abstract}
Conhecer a influência de variáveis materno-infantis e ambientais no processo desenvolvimental torna possível que atrasos sejam minimizados. Pretendeu-se comparar variáveis sociodemográficas e de desenvolvimento de bebês prematuros e a termo aos três meses de idade e identificar as influências de fatores materno-infantis associadas ao desenvolvimento destes bebês. Participaram 275 díades mãe-bebê, sendo 168 prematuros e 107 a termo. Aplicou-se uma entrevista para coleta das variáveis materno-infantis e as Escalas Bayley III para avaliar o desenvolvimento. Os resultados indicaram que há diferenças significativas entre a termo e prematuros em relação à escolaridade materna, presença de irmãos e não planejamento materno da gravidez. Prematuros apresentaram maiores atrasos no desenvolvimento cognitivo, em linguagem expressiva, motor fino e motor amplo. A identificação de atrasos aos três meses apontou para a importância de programas de estimulação precoce como fator de proteção para evitar atrasos no desenvolvimento e como orientação aos cuidadores primários do bebê.
\end{abstract}

Resumo

Palavras-chave: desenvolvimento infantil, nascimento prematuro, nascimento a termo

\section{Abstract}

Knowing the influence of maternal-infant and environmental factors in the developmental process makes it possible to minimize delays. We intended to compare socio-demographic and developmental variables of preterm and full-term babies at three months of age, and to identify the influences of maternal-infant factors associated with the development of these babies. Participants were 275 mother-baby dyads, 168 being preterm and 107 full-term babies. We applied an interview to collect maternal-infant variables and we used the Bayley III Scales to evaluate development. The results indicated that there are significant differences between full-term and preterm babies regarding maternal education, the presence of siblings, and lack of maternal pregnancy planning. Preterm babies displayed more delays in cognitive development, expressive language, fine and gross motor development. The identification of delays at three months pointed to the importance of early stimulation programs as a protective factor to avoid developmental delays and as guidance to the primary caregivers of the baby.

Keywords: child development, preterm birth, full-term birth

\footnotetext{
${ }^{1}$ Endereço de contato: Rua Jorge Ayub, n. 345, Recanto dos Nobres, Agudos, SP, CEP 17125-002. E-mail: rafaela.schiavo@gmail.com
} 


\section{Resumen}

Conocer la influencia de variables materno-infantiles y ambientales en el proceso de desarrollo hace posible que atrasos sean minimizados. Se pretendió comparar variables sociodemográficas y de desarrollo de bebés prematuros y a término a los tres meses de edad e identificar la influencia de factores materno-infantiles asociadas al desarrollo de estos bebés. Participaron 275 díadas madrebebé, siendo 168 prematuros y 107 a término. Se aplicó una entrevista para la colecta de las variables materno-infantiles y las Escalas Bayley III para evaluar el desarrollo. Los resultados indicaron que hay diferencias significativas entre bebés a término y prematuros con respecto a la escolaridad materna, presencia de hermanos y falta de planeamiento materno del embarazo. Los prematuros presentaron mayores atrasos en el desarrollo cognitivo, en lenguaje expresivo, control motor fino y grueso. La identificación de atrasos a los tres meses mostró la importancia de programas de estimulación temprana como factor de protección para evitar atrasos del desarrollo y como orientación a los cuidadores primarios del bebé.

Palabras clave: desarrollo infantil, nacimiento prematuro, nacimiento a término

\section{Introdução}

Bebês que nascem prematuramente apresentam maiores chances de risco para atrasos no desenvolvimento do que bebês que nascem a termo (Rodrigues \& Bolsoni-Silva, 2011). A imaturidade do organismo pode levar a disfunções em qualquer órgão ou sistema corporal, influenciados por fatores ambientais que podem afetar o seu desenvolvimento (Ramos \& Cumam, 2009).

A Organização Mundial da Saúde (Word Health Organization [WHO], 2018) considera nascimento prematuro aquele com menos de 37 semanas de gestação. Quanto menor for a idade gestacional do bebê, maiores as possibilidades de risco para o desenvolvimento. Algumas das condições de risco para nascimento prematuro são: gestação na adolescência (Santos et al., 2014; Martins, Santos, Sousa, Costa, \& Simões, 2011), gestantes consumidoras de drogas lícitas e ilícitas (Paula, 2018), baixo nível sociocultural, pré-eclâmpsia materna, cesáreas (Gonzaga, Santos, Silva, \& Campelo, 2016), problemas de saúde mental na gestação (Menezes et al., 2012; Bener, 2013), entre outros.

O Brasil está entre os dez países com maiores números de nascimentos prematuros, com cerca de 279.300 ocorrências por ano, segundo a Organização Mundial da Saúde (WHO, 2018). De acordo com a literatura recente, crianças prematuras têm desvantagens no desenvolvimento funcional nas áreas de autocuidado, mobilidade e função social (Lemos \& Veríssimo, 2016), nas áreas cognitiva e motora (Aljabar et al., 2014; Maggi, Magalhães, Campos, \& Bouzada, 2014; Sá et al., 2017), bem como na linguagem (Felix, Santos, \& Benítez-Burraco, 2017; Zago, Ferreira-Pinto, Ribeiro-Leite, Santos, \& Morais, 2017). Estudos apontaram também prejuízos aos prematuros no desenvolvimento motor (Maia, Silva, Oliveira, \& Cardoso, 2011) e em todas as áreas de desenvolvimento (Pinto, 2009, Rodrigues \& Bolsoni-Silva, 2011), quando comparados com os a termo.

Fatores de risco e de proteção para o desenvolvimento saudável de uma criança estão presentes nos diferentes ambientes e contextos nos quais a criança interage. Tais fatores devem ser levados em consideração ao analisar o desenvolvimento de uma criança (Zajonz, Muller \& Valentini, 2008; Maria-Mengel, \& Linhares, 2007; Tella et al., 2018). Um fator associado pode ser, também, a pouca compreensão dos cuidadores sobre a importância de propiciar estímulos ao bebê, necessários para o seu desenvolvimento (Rodrigues, 2012).

Independentemente da prematuridade, diferentes condições ambientais e biológicas podem aumentar a probabilidade de atrasos no desenvolvimento da criança ou, ainda, 
potencializar este desenvolvimento. A escolaridade, a saúde materna, as condições familiares (nível socioeconômico, boas condições de moradia, rede de apoio), se adequadas, combinam-se para prover melhores chances de sucesso (Correa, Minetto, \& Crepaldi, 2018; Tella et al., 2018). Entretanto, esses mesmos fatores, se inadequados, configuram-se como fatores de risco para o desenvolvimento. Por exemplo, a falta de cuidados pré-natais, associada à pobreza e a níveis baixos de instrução dos pais, é considerada fator relevante para geração de riscos à saúde e ao desenvolvimento do bebê (Pires, Araújo-Pedrosa, Carvalho, \& Canavarro, 2012; Fernandes, Santos Junior, \& Gualda, 2012).

Zajonz et al. (2008) alertam que fatores maturacionais adequados, quando não associados a um contexto rico em experiências, não são suficientes para possibilitar um incremento significativo no desenvolvimento da criança. Portanto, o nascimento a termo, por si só, não garante a qualidade do desenvolvimento infantil. Programas de intervenção precoce podem oferecer uma condição de proteção para evitar atrasos no desenvolvimento infantil (Carniel et al., 2017). Tais programas visam oferecer atendimento semanal, mensal ou trimestral aos bebês, avaliando o seu desenvolvimento. Quando detectado qualquer atraso, intervenções específicas são implementadas por diferentes profissionais para amenizá-lo, orientando os seus cuidadores, com o objetivo de amenizar os riscos (Sá et al., 2017). Uma pesquisa realizada por Melo e Andrade (2015) identificou que pais de bebês prematuros têm dificuldades para descrever o que é desenvolvimento normal e relacionado à prematuridade, demonstrando, assim, a importância dos programas de intervenção precoce centrado nos cuidadores.

Pesquisas têm sido elaboradas e realizadas no Brasil buscando investigar variáveis maternas e infantis de risco e proteção ao desenvolvimento. Entre as variáveis, a idade e a escolaridade materna merecem atenção. A melhor organização do ambiente, estimulação, práticas parentais mais adequadas e maiores cuidados com a saúde do bebê se relacionaram positivamente com o maior nível de escolaridade materna (Seidl-de-Moura et al., 2008; Ribas Jr., Seidl de Moura \& Bornstein, 2007; Silva \& Magalhães, 2011), enquanto filhos de mães com baixa escolaridade são inseridos em um ambiente pouco estimulador e podem ter prejuízos na capacidade de resolução de problemas, linguagem, desenvolvimento motor, memória e habilidades sociais (Tamis-Lemonda \& Rodriguez, 2011; Tella et al., 2018). Todavia, considerando o efeito da idade materna, os dados são inconclusivos. No estudo de Sartori, Saccani e Valentini (2010), os filhos de mães adolescentes tiveram desempenho motor pior do que os filhos de mães adultas, enquanto no estudo de Rodrigues, Tirelli e Silva (2012) as autoras não encontraram diferenças significativas em nenhuma das áreas avaliadas (cognição, linguagem, socialização, autocuidado e desenvolvimento motor).

Santos e Freitas (2011) apontaram que o ciclo de vida de famílias de baixa renda é marcado por inúmeras crises e suas capacidades adaptativas são frequentemente levadas além de limites humanos, resultando em prejuízos para o desenvolvimento. Quando existem riscos psicossociais e biológicos ligados, esta consequência é adicional, podendo comprometer fortemente o desenvolvimento da criança (Boyd \& Bee, 2011; Papalia \& Feldman, 2013).

Condições pré e pós-natais compõem o conjunto de variáveis que podem influenciar o desenvolvimento infantil. Entre elas, destacam-se o planejamento da gravidez, a saúde da mãe na gestação, o número de consultas pré-natais e a via de parto (Crestani et al., 2013; Pereira, Chiodelli, Rodrigues, Silva, \& Mendes, 2014; Cavalcante, Lamy Filho, França, \& Lamy, 2017). 
A via de parto também é uma variável de risco e proteção para o desenvolvimento. Os resultados obtidos por Severiano, (2017) mostraram que o parto natural influenciou positivamente o desenvolvimento de crianças até 36 meses de idade. Todavia, os dados de Cavaggioni (2017) não mostraram diferenças significativas nas áreas de desenvolvimento motor (fino e amplo), linguagem (receptiva e expressiva) e cognição, ao se comparar crianças nascidas de parto natural e de via cesárea.

Quanto às variáveis infantis, o sexo, os pesos ao nascer, a idade gestacional e a presença de síndromes e malformações são as mais pesquisadas como aquelas que influenciam o desenvolvimento. Pereira et al. (2014) encontraram, nas áreas avaliadas pelo Inventário Portage Operacionalizado, melhor desempenho em cognição para os bebês com maior peso ao nascer e melhor desempenho em desenvolvimento motor para os bebês com maior idade gestacional. Maia et al. (2011) compararam o desenvolvimento motor de crianças prematuras e a termo e encontraram melhor desempenho para as crianças nascidas a termo. Os achados de Pessoa, Martins, Lima e Gaiva (2015) confirmam tais resultados. Eles encontraram que a prematuridade e o baixo peso são fatores que resultam em prejuízo para o desenvolvimento infantil. Quanto ao sexo, os estudos têm apontado para diferenças entre meninos e meninas. Almeida, Rodrigues e Salgado (2012) encontraram resultados que apontaram que as meninas entre três e seis meses de vida tiveram desempenho melhor que os meninos.

Há diferenças entre o desenvolvimento de bebês a termo e prematuros, quando se comparam as idades cronológicas (Maia et al., 2011; Isotani, Azevedo, Chiari, \& Perissinoto, 2009). Tais dados reforçam a importância de estudos em idades precoces, o que permitiria que profissionais da saúde elaborassem ações preventivas para atender esta população. Tão importante quanto, seria identificar fatores associados ao desenvolvimento de bebês, prematuros e a termo, que podem ser alvo de políticas públicas mais amplas enquanto prevenção primária para o adequado desenvolvimento infantil.

O presente estudo teve como objetivo comparar variáveis sociodemográficas e de desenvolvimento de bebês a termo e prematuros aos três meses de idade e identificar a influência de fatores materno-infantis associados ao desenvolvimento de bebês a termo e prematuros.

\section{Método}

\section{Aspectos Éticos da Pesquisa}

Este projeto de pesquisa faz parte do projeto "Variáveis maternas e do bebê: correlação entre interação e desenvolvimento infantil" (Processo FAPESP n. 2016/11.557-4) e foi aprovado pelo Comitê de Ética da Faculdade de Ciências da UNESP, campus de Bauru (Processo CAAE: 64920817.9.0000.5398). Todas as providências cabíveis foram tomadas por ocasião do convite para a participação neste projeto, de acordo com a resolução do CONEP. A partir do aceite e redimidas todas as dúvidas, as participantes ou seus responsáveis assinaram o Termo de Consentimento Livre e Esclarecido.

\section{Participantes}

Participaram 275 díades mãe-bebê, cujos bebês estavam com três meses de idade e frequentavam o projeto de extensão "Acompanhamento do desenvolvimento de bebês: 
avaliação e orientação aos pais", que funciona no Centro de Psicologia Aplicada (CPA) da UNESP, campus de Bauru, e na SORRI - Bauru. Destes, 107 eram bebês a termo e 168 prematuros.

Considerando a amostra total, a maioria das participantes desta pesquisa tinha idade acima de 20 anos (89\%), com mais de oito anos de estudo (82\%). Delas, metade (50\%) era mãe de pelo menos um filho e um pouco mais da metade (58\%) não planejou a gravidez. Quanto às características dos bebês, metade (50\%) era do sexo masculino, a maioria nasceu prematuramente (61\%) e quase metade deles com peso superior A $2.500 \mathrm{~g}(49 \%)$. A maioria nasceu via cirurgia cesariana (74\%). Quanto ao atraso no desenvolvimento, a área mais afetada foi a cognição (70\%), seguida do motor amplo (63\%), linguagem receptiva (51\%), expressiva (45\%), e a área menos afetada foi a de motor fino (36\%).

\section{Materiais}

Para a investigação dos dados sociodemográficos da mãe, foi aplicada uma entrevista que visava identificar as características maternas de idade, escolaridade, número de filhos e planejamento da gestação; e, sobre o bebê, as características de sexo, idade gestacional de nascimento, peso ao nascimento e via de nascimento.

Para avaliação do desenvolvimento infantil, foi utilizada a Bayley Scales of Infant Development-III - BSID-III (Bayley, 2006), traduzida no Brasil como Bayley III, Escalas de Desenvolvimento do Bebê e da Criança Pequena (Bayley, 2018). Esta escala avalia o desenvolvimento funcional progressivo de crianças de um a 42 meses de idade. É dividida em cinco escalas: Escala Cognitiva (avalia o desenvolvimento sensório motor, exploração e manipulação, formação de conceitos, memória e outros processos cognitivos); Escala de Linguagem (avalia comunicação receptiva e expressiva em duas subescalas distintas); Escala Motora (avalia habilidades motoras em geral e motricidade fina); Escala Socioemocional (identifica os principais marcos do desenvolvimento social e emocional); Escala do Comportamento Adaptativo (habilidades funcionais do cotidiano). Para este estudo, utilizaram-se os dados ponderados considerando a idade cronológica dos bebês das escalas Cognitiva, de Linguagem (Receptiva e Expressiva) e Motora (Ampla e Fina).

\section{Local}

Os dados de desenvolvimento infantil foram coletados em salas de atendimento para bebês, disponíveis no Centro de Psicologia Aplicada (CPA) da Faculdade de Ciências da UNESP e na SORRI, ambas as instituições de Bauru, estado de São Paulo (SP). Nelas estão disponíveis: mesa, cadeiras, cama alta e armário para acondicionamento de materiais.

\section{Procedimento de coleta de dados}

Os bebês e suas mães foram identificados em dois projetos de extensão que acompanham o desenvolvimento de bebês em condições de risco e não risco (CPA) e prematuros, na SORRI. Por ocasião do convite para a sua participação na pesquisa, foram cumpridos os trâmites éticos. Em ambos os projetos, os bebês a partir de três meses de idade têm o desenvolvimento monitorado. Durante o primeiro ano de vida, são avaliados trimestralmente. 
Nas duas instituições, a família recebe orientações e atendimento interdisciplinar, no caso de prematuros com menos de 35 semanas de gestação, na SORRI.

Para a primeira avaliação do desenvolvimento do bebê, aos três meses, eram agendados dia e horário com as mães que participavam dos projetos de extensão. Inicialmente, para a coleta de dados sociodemográficos, era realizada uma entrevista com a mãe e, em seguida, a avaliação do desenvolvimento dos bebês, feita por meio das Escalas Bayley III. A mãe do bebê identificado com atraso no desenvolvimento recebia orientações pertinentes e, no caso de prematuro, na SORRI, este era encaminhado para atendimentos com profissionais especializados, em atendimento interdisciplinar.

\section{Procedimento de análise de dados}

Para as análises de desenvolvimento, obtidas com a BSID-III, optou-se por utilizar a pontuação ponderada alcançada nos itens referentes à idade cronológica dos bebês nascidos a termo e prematuros. Procedeu-se a correção seguindo as normas especificas do Manual. Os dados foram analisados com o programa Statistical Package for the Social Sciences (SPSS, versão 21.0; Chicago INc II, USA). Antes de proceder à análise estatística inferencial, foi aplicado o teste de Shapiro Wilk, para testar a normalidade das variáveis. O teste Qui-Quadrado foi realizado para comparar as variáveis entre bebês prematuros e a termo, e o teste de Regressão Logística foi utilizado para identificar os fatores associados ao desenvolvimento. Para esta análise, foram binarizadas todas as variáveis numéricas e categóricas. O nível de significância adotado foi de $p<0,05$.

\section{Resultados}

A Tabela 1 mostra os dados de comparação das variáveis sociodemográficas entre as díades mãe-bebê nascidos a termo e prematuros. Nota-se que houve diferenças significativas entre os bebês nascidos a termo e prematuros, sendo mais frequentes entre os prematuros as mães com baixa escolaridade, com mais de um filho e que não planejaram a gestação. Quanto às características dos bebês, nota-se que os prematuros nasceram com menor peso, apresentaram maior proporção de atraso no desenvolvimento cognitivo, linguagem expressiva, motor fino e motor amplo em relação aos bebês nascidos a termo.

Tabela 1

Comparação de variáveis da díade mãe-bebê entre prematuros e a termo ( $N=275)$

\begin{tabular}{|c|c|c|c|c|c|}
\hline & \multicolumn{2}{|c|}{ A termo $(n=107)$} & \multicolumn{2}{|c|}{ Prematuro $(n=168)$} & \multirow{2}{*}{ p-valor } \\
\hline & FA (n) & $\mathrm{FR}(\%)$ & $F A(n)$ & $\mathrm{FR}(\%)$ & \\
\hline \multicolumn{6}{|l|}{ Características maternas } \\
\hline Escolaridade & & & & & 0,000 \\
\hline Até 9 anos de estudo & 8 & 16 & 43 & 84 & \\
\hline Acima de 9 anos de estudo & 99 & 44 & 125 & 56 & \\
\hline Paridade & & & & & 0,013 \\
\hline Primípara & 63 & 46 & 73 & 54 & \\
\hline Multípara & 44 & 32 & 95 & 68 & \\
\hline Planejamento da gravidez & & & & & 0,002 \\
\hline Sim & 57 & 50 & 58 & 50 & \\
\hline
\end{tabular}




\begin{tabular}{|c|c|c|c|c|c|}
\hline & \multicolumn{2}{|c|}{ A termo $(n=107)$} & \multicolumn{2}{|c|}{ Prematuro $(n=168)$} & \multirow{2}{*}{ p-valor } \\
\hline & $\mathrm{FA}(\mathrm{n})$ & FR (\%) & $F A(n)$ & $\mathrm{FR}(\%)$ & \\
\hline Não & 50 & 31 & 110 & 69 & \\
\hline \multicolumn{6}{|l|}{ Características do bebê } \\
\hline Peso ao nascimento & & & & & 0.000 \\
\hline Normal & 104 & 76 & 32 & 24 & \\
\hline Baixo peso & 3 & 2 & 136 & 98 & \\
\hline \multicolumn{6}{|c|}{ Áreas do desenvolvimento } \\
\hline Cognição & & & & & 0,001 \\
\hline Normal & 45 & 54 & 38 & 46 & \\
\hline Atraso & 62 & 32 & 130 & 68 & \\
\hline Linguagem receptiva & & & & & 0,109 \\
\hline Normal & 59 & 44 & 76 & 56 & \\
\hline Atraso & 48 & 34 & 92 & 66 & \\
\hline Linguagem expressiva & & & & & 0,040 \\
\hline Normal & 40 & 32 & 84 & 68 & \\
\hline Atraso & 67 & 44 & 84 & 56 & \\
\hline Motor fino & & & & & 0,000 \\
\hline Normal & 83 & 47 & 93 & 53 & \\
\hline Atraso & 24 & 24 & 75 & 76 & \\
\hline Motor amplo & & & & & 0,000 \\
\hline Normal & 62 & 61 & 40 & 39 & \\
\hline Atraso & 45 & 26 & 128 & 74 & \\
\hline
\end{tabular}

Teste Qui-Quadrado; FA: Frequência Absoluta; FR: Porcentagem.

Considerando o total da amostra, os dados apresentados na Tabela 2 indicam que não foram encontrados fatores maternos ou dos bebês associados ao atraso nas áreas do desenvolvimento cognitivo, linguagem expressiva e receptiva. Entretanto, encontrou-se que o atraso no desenvolvimento motor fino está associado ao nascimento prematuro e que ser menino diminuiu as chances de atrasos nesta área aos três meses de idade. $\mathrm{O}$ atraso na área do desenvolvimento motor amplo foi associado também à prematuridade, aumentando as chances de atraso nesta área do desenvolvimento aos três meses de idade.

Tabela 2

Fatores associados ao atraso no desenvolvimento de bebês aos três meses de idade $(N=275)$

\begin{tabular}{lccc}
\hline \multicolumn{1}{c}{ Desenvolvimento } & p-valor & OR & IC-95\% \\
\hline Motor fino & & & \\
\hline Prematuridade & 0,005 & 3,448 & $1,677-7,089$ \\
Sexo masculino & 0,007 & 0,480 & $0,306-0,753$ \\
\hline Motor amplo & & & \\
\hline Prematuridade & 0,001 & 4,014 & $1,956-8,237$ \\
\hline
\end{tabular}

Teste de Regressão Logística; p-valor p < 0,05; OR: Odds Ratio; IC-95\%: Intervalo de Confiança de 95\%.

Nota-se na Tabela 3, quando realizada a análise separada dos dois grupos de bebês, a termo e prematuros, a respeito dos fatores associados ao desenvolvimento, que, entre os bebês a termo, somente a área de desenvolvimento motor amplo mostrou associação com 
a variável número de filhos, sendo que há menores chances de atraso nesta área aos três meses de vida, quando o bebê tem irmãos. E, entre os bebês prematuros, nascer com baixo peso aumenta as chances de apresentar atrasos no desenvolvimento cognitivo aos três meses. Observa-se, também, que nascer menino diminuiu as chances para atraso na área de desenvolvimento motor fino e que ter irmãos aumentou as chances de atraso nesta área aos três meses.

Tabela 3

Fatores associados ao atraso no desenvolvimento de bebês a termo $(n=107) e$ prematuros $(n=168)$

\begin{tabular}{|c|c|c|c|}
\hline Desenvolvimento & p-valor & OR & IC-95\% \\
\hline Bebês nascidos a termo & & & \\
\hline $\begin{array}{l}\text { Motor amplo } \\
\text { Ter irmãos }\end{array}$ & 0,043 & 0,410 & $0,199-0,848$ \\
\hline Bebês nascidos prematuros & & & \\
\hline $\begin{array}{l}\text { Cognitivo } \\
\text { Baixo peso }\end{array}$ & 0,025 & 2,710 & $1,302-5,639$ \\
\hline $\begin{array}{l}\text { Motor fino } \\
\text { Masculino }\end{array}$ & 0,041 & 0,503 & $0,290-0,874$ \\
\hline Ter irmãos & 0,05 & 1,986 & $1,094-3,604$ \\
\hline
\end{tabular}

\section{Discussão}

Os resultados indicaram que uma proporção maior de bebês que nasceram prematuros eram filhos de mães com baixa escolaridade. Não raro, a baixa escolaridade é associada a vários fatores de risco à saúde materno-infantil (Zajonz et al., 2008; Santos et al., 2009). Mães com menor escolaridade têm mais desvantagens sociais e materiais, piores níveis de informação e menor possibilidade de emprego estável, o que pode influir na perspectiva de futuro e de qualidade de vida do bebê (Seidl-de-Moura et al., 2008; Ribas Jr. et al., 2007; Silva \& Magalhães, 2011; Tella et al., 2018).

Fatores como organização do ambiente, expectativas e práticas parentais, experiências com materiais para estimulação cognitiva e variação da estimulação diária estariam mais presentes em mães com maior escolaridade e, também, teriam íntima relação com a melhor utilização da renda no que diz respeito aos cuidados infantis e dos serviços públicos que estiverem ao alcance das famílias (Tella et al., 2018). Estas mães realizariam com mais propriedade os cuidados preventivos e curativos com as crianças.

Silva et al. (2011) concluíram, a partir de um estudo de revisão, que o desenvolvimento infantil é significativamente influenciado pela escolaridade materna, além do nível socioeconômico, pois a renda per capita familiar tem impacto, direta ou indiretamente, no desenvolvimento infantil. Mães com mais de um filho também tiveram uma maior proporção de filhos prematuros. A literatura tem indicado que um dos fatores de risco para nascimento prematuro é a alta ansiedade materna na gestação (Alder, Fink, Bitzer, Hösli, \& Holzgreve, 2007; Bener, 2013; Conde \& Figueiredo, 2003), e a alta ansiedade materna está associada 
à baixa escolaridade e às dificuldades socioeconômicas da mãe (Simas, Souza, \& ScorsoliniComin, 2013; Schiavo, Rodrigues, \& Perosa, 2018).

Um estudo realizado por Schiavo et al. (2018) investigou 479 gestantes comparando ansiedade de primigestas e multigestas no terceiro trimestre gestacional. Elas identificaram que $38 \%$ das primigestas apresentavam alta ansiedade e $62 \%$ das multigestas apresentavam alta ansiedade. As autoras justificam a maior prevalência em multigestas pelo fato de que o nascimento de mais um filho pode aumentar as preocupações financeira, ocupacional, conjugal e familiar, tendo que reconfigurar todos esses sistemas com possíveis prejuízos em sua perspectiva de futuro, gerando incertezas e dificuldades. Desta forma, é possível inferir que mulheres com baixa escolaridade e com a presença de mais um filho podem ter aumento de preocupações diárias com alimentação, contas a pagar, interferências na relação conjugal e na maternidade de toda a prole, configurando-se em um contexto de vida que pode explicar por que a mães de mais de um filho foram mais suscetíveis de terem parto prematuro.

O não planejar a gestação também aumentou a proporção de mães com parto prematuro. Seguindo o mesmo raciocínio anterior sobre a ansiedade gestacional, a literatura aponta que o não planejamento da gravidez aumenta as chances de a mulher apresentar alta ansiedade na gestação (Benute et al., 2010; Schiavo et al., 2018) e, como já mencionado, a alta ansiedade gestacional pode ser uma das variáveis envolvidas no parto prematuro (Alder et al., 2007; Bener, 2013; Conde \& Figueiredo, 2003). Como descrito, bebês prematuros apresentaram maior índice de nascimento com baixo peso. Pesquisas têm associado o baixo peso do bebê ao nascimento prematuro (Carniel, Zanolli, Antonio, \& Morcillo, 2008; Araújo \& Tanaka, 2007). O feto atinge peso acima de 2.500 gramas geralmente por volta da 36a semana de gestação, fato que justifica o baixo peso da maioria dos bebês que nasceram prematuros neste estudo, ou seja, antes de completar 37 semanas.

Outro resultado que corrobora dados anteriores foi o de que bebês prematuros apresentaram maior proporção de atrasos no desenvolvimento quando comparados aos bebês a termo (Maia et al., 2011; Rodrigues \& Bolsoni-Silva, 2011, Isotani et al., 2009, Pinto, 2009). Neste estudo, não foi utilizada a idade corrigida para avaliar o desenvolvimento de bebês prematuros, justamente para verificar se influências sociodemográficas maternas e dos bebês poderiam interferir no desenvolvimento. Entretanto, fica claro neste estudo que, aos três meses de vida, o bebê prematuro ainda não teve oportunidade de receber muitos estímulos do ambiente como um bebê a termo. Em geral, os bebês prematuros necessitam de internação e cuidados hospitalares, e a estimulação que ele recebe nos três primeiros meses pode ser diferente daquela do bebê que nasceu em boas condições, que não necessitou dos mesmos cuidados especiais. Isso reforça a ideia de que, ao avaliar o desenvolvimento do bebê prematuro, pode ser interessante levar em conta, também, a sua idade corrigida (Saccani, Zanella, Notari, \& Valentini, 2017).

Neste estudo, observou-se que, ao comparar o desenvolvimento de bebês prematuros e a termo, as áreas que apresentaram diferenças entre os comportamentos aos três meses foram cognição, linguagem expressiva, motor fino e motor amplo. A linguagem receptiva não se mostrou diferente aos três meses, quando comparados bebês nascidos prematuros e a termo, confirmando a prontidão de que os bebês, ainda que prematuros, mantêm atenção às informações que recebem do meio (Gerhardt, 2017; Boyd \& Bee, 2011). Todavia, autores têm apontado para prejuízos na linguagem de bebês prematuros em idades posteriores aos 
três meses (Felix et al., 2017; Zago et al., 2017). Em relação ao atraso na linguagem, fatores como dificuldades no aleitamento materno de crianças prematuras podem influenciar na flacidez de lábios, língua e bochecha, intervindo, assim, negativamente no adequado desenvolvimento da linguagem (Brusco \& Delgado, 2014). De acordo com Lopes, Tani e Maía, (2011), os riscos para atrasos no desenvolvimento de crianças prematuras podem estar relacionados a: dificuldades precoces e prolongadas; nascer com órgãos imaturos; necessidade de tratamento em unidades de cuidados intensivos, causando estresse e falta de experiências sociais com o cuidador familiar.

Neste estudo, fatores maternos como idade da mãe, estado civil, número de filhos e planejamento da gestação não se associaram ao atraso no desenvolvimento do bebê aos três meses de idade, não confirmando dados de estudos similares (Sartori et al., 2010; Crestani et al., 2013; Pereira et al., 2014; Cavalcanti et al., 2017). No entanto, o nascimento prematuro se mostrou um fator associado a atrasos no desenvolvimento aos três meses nas áreas de motricidade fina e ampla, resultado esse já encontrado também na literatura (Kreling, Brito, \& Matuso, 2006). O desenvolvimento motor se refere ao processo de progressão dos movimentos simples e desorganizados para habilidades motoras organizadas e complexas (Papalia \& Feldman, 2013). O desenvolvimento motor atípico não se configura, necessariamente, por alterações neurológicas ou estruturais (Willrich, Azevedo, \& Fernandes, 2004), mas pode estar associado à pouca estimulação, devido a fatores de ordem ambiental, como a baixa escolaridade dos pais e condições socioeconômicas ruins (Zajonz et al., 2008).

Um dado interessante é o de que nascer menino diminuiu as chances de atraso na área de motricidade fina, indicando que mães de meninos podem estimular mais o seu bebê ou permitem que os bebês explorem mais o ambiente em relação às mães de meninas. Tais dados diferem dos encontrados por Formiga e Linhares (2009) e Almeida et al. (2012), cujos desempenhos das meninas nas tarefas de desenvolvimento superaram o dos meninos. Este estudo indicou também que bebês nascidos a termo com irmãos têm menor chance de apresentar atraso aos três meses na área de motricidade ampla. Uma hipótese é de que é possível que mães de mais de um filho adquiram habilidades com os cuidados com o bebê, executando comportamentos que permitam maior liberdade e autonomia à criança e possibilitando, assim, um ambiente mais propício para exploração.

Já o inverso ocorre para os prematuros, pois, quando estes têm irmãos, aumentam-se as chances de atraso na área motora fina aos três meses. Da mesma forma que a mãe de um bebê nascido a termo permite que a criança tenha mais autonomia quando os cuidados com o bebê não são mais uma novidade, ela também pode agir do mesmo modo com o bebê prematuro, não atenta aos atrasos de desenvolvimento. Entretanto, o bebê prematuro necessita de cuidados mais intensos, precisando receber mais estimulação do seu meio, orientado por especialistas, o que justifica sua inserção precoce em serviços de intervenção.

Este dado reforça a importância de oportunizar a troca de informações e experiências sobre o desenvolvimento infantil, mesmo quando o bebê não tem risco para o desenvolvimento previamente identificado. Ribeiro, Perosa e Padovani (2014) sugerem que programas direcionados à criança de risco são importantes para aumentar as suas chances de sucesso. Pereira et al. (2014) enfatizam a importância de programas de intervenção precoce, reconhecendo que quem mais precisa não tem, provavelmente, acesso a eles. 
Bebês prematuros que nascem com outra variável de risco, que é o baixo peso, têm maiores chances de atraso no desenvolvimento cognitivo aos três meses de vida. Outros estudos já apontaram também atrasos na área cognitiva de bebês que nasceram prematuros com baixo peso (Maggi et al., 2014; Sá et al., 2017). Para Aljabar et al. (2014), a alta prevalência de atrasos cognitivos em bebês prematuros pode ser explicada pelo fato de bebês com idade gestacional inferior a 30 semanas não apresentarem conectividade das redes neuronais de forma tão eficiente quanto os bebês com mais de 30 semanas gestacionais. Embora a organização cerebral para as conectividades permaneça intacta em bebês prematuros, permitindo funções neurológicas complexas, a exposição prematura ao ambiente extrauterino está associada à arquitetura de rede alterada e à redução da capacidade da rede.

\section{Considerações Finais}

O presente estudo teve como objetivo comparar variáveis sociodemográficas e de desenvolvimento de bebês a termo e prematuros aos três meses de idade e identificar a influência de fatores materno-infantis associados ao desenvolvimento de bebês a termo e prematuros. Os dados obtidos mostram o prejuízo da prematuridade para o desenvolvimento, mas reforçam, também, as considerações de diversos estudos na área, que destacaram que as variáveis sociodemográficas não devem ser encaradas isoladamente. É necessário que se investigue mais sobre condições socioeconômicas, ambientais e qualidade de cuidados e de estimulação presentes no ambiente da criança. O ambiente familiar é um dos contribuintes potenciais para o desenvolvimento infantil enriquecido por orientações de especialistas em desenvolvimento infantil, garantindo cuidadores bem informados, fruto de oportunidades de conhecimento e de reflexões sobre temas de necessidade dos bebês, especialmente os prematuros.

Entre as limitações do presente estudo, destaca-se o número desigual de bebês a termo e prematuros, o que poderia dar mais consistência aos dados. Também, o tipo de análise conduzida é mais confiável com amostras maiores do que a utilizada no presente estudo. Outra limitação se refere à ausência de dados sobre saúde emocional materna, um dado que tem sido associado a outras variáveis sociodemográficas, auxiliando na explicação de atrasos no desenvolvimento infantil. Sugerem-se, então, estudos com populações equivalentes e maiores e a coleta de dados sobre saúde emocional materna para a explicação dos desfechos de desenvolvimento, tanto para bebês nascidos a termo como prematuros.

Reforça-se que se deve considerar o contexto e a situação de cada família, salientando-se a importância do crescimento de pesquisas na área do desenvolvimento infantil e da saúde, bem como na elaboração de políticas públicas necessárias e condizentes com a situação das famílias brasileiras, de forma a propiciar, antes de tudo, o suporte necessário em saúde, alimentação e educação. Um trabalho integrado entre a atenção primária, secundária e terciária deve existir para que nossas crianças e nossas famílias se desenvolvam da forma mais saudável e mais feliz.

\section{Referências}

Alder, J., Fink, N., Bitzer, J., Hösli, I., \& Holzgreve, W. (2007). Depression and anxiety during pregnancy: A risk factor for obstetric, fetal and neonatal outcome? A critical of the 
literature. Journal of Maternal-Fetal \& Neonatal Medicine, 20(3), 189-209. Recuperado de http://informahealthcare.com/doi/abs/10.1080/14767050701209560

Aljabar, G. B. P., Zebari, S., Tusor, N., Arichi, T., Merchant, N., Robinson, E. C., Ogundipe, E., Rueckert, D., Edwards, A. D., \& Counsell, S. J. (2014). Rich-club organization of the newborn human brain. PNAS, 111(20), 7456-7461. Recuperado de https://doi. org/10.1073/pnas.1324118111

Almeida, C. G. M., Rodrigues, O. M. P. R., \& Salgado, M. H. (2012). Diferenças no desenvolvimento de meninos e meninas em condições de risco. Boletim de Psicologia, 62(136), 1-14. Acessado de: http://pepsic.bvsalud.org/pdf/bolpsi/v62n136/v62n136a02.pdf

Araújo, B. F., \& Tanaka, A. C. d’A. (2007). Fatores de risco associados ao nascimento de recém-nascidos de muito baixo peso em uma população de baixa renda. Caderno de Saúde Pública, 23(12), 2869-2877. Recuperado de http://dx.doi.org/10.1590/ S0102-311X2007001200008

Bayley, N. (2006). Bayley scales of infant and toddler development (3a ed.). New York: PsychCorp.

Bayley, N. (2018). Bayley III: Escalas de desenvolvimento do bebê e da criança pequena. São Paulo: Pearson Clinical Brasil.

Bener, A. (2013). Psychological distress among mothers of preterm infants and associated factors: A neglected public health problem. Revista Brasileira de Psiquiatria, 35(3), 231236. doi: http://dx.doi.org/10.1590/1516-4446-2012-0821

Benute, G. R. G., Nomura, R. M. Y., Reis, J. S., Fraguas Junior, R., Lucia, M. C. S., \& Zugaib, M. (2010). Depression during pregnancy in women with a medical disorder: Risk factors and perinatal outcomes. Clinics, 65(11), 1127-1131. Recuperado de: http://www.scielo. br/scielo.php?pid=S1807-59322010001100013\&script=sci_arttext

Boyd, D., \& Bee, H. (2011). A criança em desenvolvimento. Porto Alegre: Artmed.

Brusco, T. R., \& Delgado, S. E. (2014). Caracterização do desenvolvimento da alimentação de crianças nascidas pré-termo entre três e 12 meses. Revista CEFAC., 16(3), 917-928. doi: http://dx.doi.org/10.1590/1982-021620145313.

Carniel, E. F., Zanolli, M. L., Antonio, M. A. R. G. M., \& Morcillo, A. M. (2008). Determinantes do baixo peso ao nascer a partir das declarações de nascidos vivos. Revista Brasileira de Epidemiologia, 11(1), 169-179. doi: http://dx.doi.org/10.1590/ S1415-790X2008000100016

Carniel, C. Z., Furtado, M. C. C., Vicente, J. B., Abreu, R. Z., Tarozzo, R. M., Cardia, S. E. T. R., Massei, M. C. I., \& Cerveira, R. C. G. F. (2017). Influência de fatores de risco sobre o desenvolvimento da linguagem e contribuições da estimulação precoce: Revisão integrativa da literatura. Revista CEFAC, 19(1), 109-118. doi: http://dx.doi.org/10.1590/1982-0216201719115616 Cavaggioni, A. P. M. (2017). Influência da via de parto no desenvolvimento infantil: Comparação por meio da Escala Bayley III (Dissertação de Mestrado, Universidade Metodista de São Paulo [UMESP], São Bernardo do Campo, SP).

Cavalcante, M. C. V., Lamy Filho, F., França, A. K. T. C., \& Lamy, Z. C. (2017). Relação Mãefilho e fatores associados: Análise hierarquizada de base populacional em uma capital do Brasil-Estudo BRISA. Ciência \& Saúde Coletiva, 22(5), 1683-1693. doi: http://dx.doi. org/10.1590/1413-81232017225.21722015 
Conde, A., \& Figueiredo, B. (2003). Ansiedade na gravidez: fatores de risco e implicações para a saúde e bem-estar da mãe. Revista de Psiquiatria Clínica, 24(3), 197-209. Recuperado de: http://repositorium.sdum.uminho.pt/bitstream/1822/4217/1/Ansiedade\%20na\%20 gravidez\%20\%282003\%29.pdf

Correa, W., Minetto, M. F., \& Crepaldi, M. A. (2018). Família como promotora do desenvolvimento de crianças que apresentam atrasos. Pensando Família, 22(1), 44-58. Recuperado de: http://pepsic.bvsalud.org/pdf/penf/v22n1/v22n1a05.pdf.

Crestani, A. E., Mattana, F., Moraes, A. H. B., \& Souza, A. P. R. (2013). Fatores socioeconômicos, obstétricos, demográficos e psicossociais como risco ao desenvolvimento infantil. Revista CEFAC 15(4), 847-856. Recuperado de: http://www.scielo.br/pdf/rcefac/v15n4/12.pdf

Felix, J., Santos, M. E., \& Benítez-Burraco, A. (2017). Linguagem espontânea de crianças prematuras aos 4 e 5 anos de idade. Revista CEFAC, 19(6), 742-748. doi: https://doi. org/10.1016/j.jpedp.2013.10.004

Fernandes, A. O., Santos Junior, H. P. O., \& Gualda, D. M. R. (2012). Gravidez na adolescência: Percepções das mães de gestantes jovens. Acta Paulista de Enfermagem, 25(1), 55-60. doi: http://dx.doi.org/10.1590/S0103-21002012000100010

Formiga, C. K. M. R., \& Linhares, M. B. M. (2009). Avaliação do desenvolvimento inicial de crianças nascidas pré-termo. Revista Escola de Enfermagem USP., 43 (2), 472-480. doi: http://dx.doi.org/10.1590/S0080-62342009000200030

Gerhardt, S. (2017). Porque o amor é importante: Como o afeto molda o cérebro do bebê. Porto Alegre: Artmed.

Gonzaga, I. C. A. Santos, S. L. D., Silva, A. R. V., \& Campelo, V. (2016). Atenção pré-natal e fatores de risco associados à prematuridade e baixo peso ao nascer em capital do nordeste brasileiro. Ciência \& Saúde Coletiva, 21(6), 1965-1974. doi: http://dx.doi-org. ez67.periodicos.capes.gov.br/10.1590/1413-81232015216.06162015

Isotani, S. M., Azevedo, M. F., Chiari, B. M., \& Perissinoto, J. (2009). Linguagem expressiva de crianças nascidas pré-termo e termo aos dois anos de idade. Pró-Fono Revista de Atualização Cientifica, 21(2), 155-160. doi: http://dx.doi.org/10.1590/S0104-56872009000200012

Kreling, K. C. A, Brito, A. S. J., \& Matuso, T. (2006). Fatores perinatais associados ao desenvolvimento neuropsicomotor de recém-nascidos de muito baixo peso. Pediatria (São Paulo), 28(2), 98-108. Recuperado de: http://ucbweb2.castelobranco.br/webcaf/ arquivos/13150/5117/Fatores_perinatais_e_desenvolvimento_motor_do_RN_de_ muito_baixo_peso.pdf

Lemos, R. A., \& Veríssimo, M. Ó. R. (2016). Functional development of preterm infants: An integrative literature review. Fisioterapia em Movimento, 29(3), 623-633. doi: http:// dx.doi.org/10.1590/1980-5918.029.003.AO20

Lopes, A. A. T., Tani, G., \& Maía, J. A. R. (2011). Desempenho neuromotor, prematuridade e baixo peso à nascença. Revista Brasileira de Cineantropometria e Desempenho Humano, 13(1), 73-81. doi: http://dx.doi.org/10.5007/1980-0037.2011v13n1p73

Maggi, E. F., Magalhães, L. C., Campos, A. F., \& Bouzada, M. C. F. (2014). Preterm children have unfavorable motor, cognitive, and functional performance when compared to term children of preschool age. Jornal de Pediatria, 90(4), 377-383. doi: https://doi. org/10.1016/j.jpedp.2013.10.004 
Maia, P. C., Silva, L. P., Oliveira, M. M. C., \& Cardoso, M. V. L. M. L. (2011). Desenvolvimento motor de crianças prematuras e a termo - Uso da Alberta Infant Motor Scale. Acta Paulista de Enfermagem, 24(5), 670-675.

Maria-Mengel, M. R. S., \& Linhares, M. B. M. (2007). Risk factors for infant developmental problems. Revista Latino Americana de Enfermagem, 15(spe), 837-842. doi: http://dx.doi. org/10.1590/S0104-11692007000700019

Martins, M. G., Santos, G. H. N., Sousa, M. S., Costa, J. E. F. B., \& Simões, V. M. F. (2011). Associação de gravidez na adolescência e prematuridade. Revista Brasileira de Ginecologiae Obstetrícia, 33(11), 354-360. doi: http://dx.doi.org/10.1590/S0100-72032011001100006

Melo, M. R. O., \& Andrade, I. S. N. S. (2015). Child development and prematurity: A reflection on maternal knowledge and expectations. Brazilian Journal in Health Promotion, 26(4), 548-553. Recuperado de: https://www.redalyc.org/pdf/408/40831096013.pdf

Menezes, L. O., Pinheiro, R. T., Quevedo, L. A., Oliveira, S. S., Silva, R. A., Pinheiro, K. A. T., Santo, G. C. E., \& Jansen, K. (2012). O impacto do baixo peso ao nascer relacionado à depressão gestacional para o financiamento federal da saúde pública: Uma análise do Município de Pelotas, Rio Grande do Sul. Caderno de Saúde Pública, 28(10), 1939-1948. doi: http://dx.doi.org/10.1590/S0102-311X2012001000012

Papalia, D. E., \& Feldman, R. D. (2013). Desenvolvimento Humano (12a ed.). Porto Alegre: AMGH.

Paula, R. S. K. (2018). Consequências à criança do uso de drogas durante a gestação: Um artigo de revisão. Directory of Open Access Journals, 58(1), 45-52. doi: http://dx.doi. org/10.20513/2447-6595.2018v58n1p45-52

Pereira, V. A., Chiodelli T., Rodrigues, O. M. P. R., Silva, C. S. O., \& Mendes, V. F. (2014). Desenvolvimento do bebê nos dois primeiros meses de vida: Variáveis maternas e sociodemográficas. Pensando Família 18(1), 64-77. Recuperado de: http://pepsic.bvsalud. org/pdf/penf/v18n1/v18n1a07.pdf

Pessoa, T. A. O., Martins, C. B. G., Lima, F. C. A. L. \& Gaíva, M. A. M. (2015). O crescimento e desenvolvimento frente à prematuridade e baixo peso ao nascer. Avances en Enfermería, 33(3), 401-411. doi:http://dx.doi.org/10.15446/av.enferm.v33n3.44425

Pinto, E. B. (2009). O desenvolvimento do comportamento do bebê prematuro no primeiro ano de vida. Psicologia: Reflexão e Crítica, 22(1), 76-85. doi: https://dx.doi.org/10.1590/ S0102-79722009000100011

Pires, R., Araújo-Pedrosa, A., Carvalho, P., \& Canavarro, M. C. (2012). Necessidades emergentes de promoção da saúde na transição para a parentalidade: Variáveis relacionais de risco para a depressão durante a gravidez na adolescência. In J.L.P. Ribeiro, I. Leal, A. Pereira, \& S. Monteiro (Orgs.), Psicologia da saúde: Desafios à promoção da saúde em doenças crónicas (pp. 250-256). Aveiro: Placebo Editora.

Ramos, H. A. C., \& Cumam, R. K. N. (2009). Fatores de risco para prematuridade: Pesquisa documental. Escola Anna Nery Revista de Enfermagem, 13(12), 297-304. doi: http:// dx.doi.org/10.1590/S1414-81452009000200009

Ribas Jr., R. C., Seidl de Moura, M. L. S., \& Bornstein, M. H. (2007). Cognições maternas acerca da maternidade e do desenvolvimento humano: Uma contribuição ao estudo da psicologia parental. Revista Brasileira de Crescimento e Desenvolvimento Humano, 17(1), 104-113. Recuperado de: http://pepsic.bvsalud.org/pdf/rbcdh/v17n1/10.pdf 
Ribeiro, D. G., Perosa, G. B., \& Padovani, F. H. P. (2014). Fatores de risco para o desenvolvimento de crianças atendidas em Unidades de Saúde da Família, ao final do primeiro ano de vida. Ciência e Saúde Coletiva, 19(1), 215-226. Recuperado de: http://www.scielo.br/pdf/csc/ v19n1/1413-8123-csc-19-01-00215.pdf

Rodrigues, O. M. P. R. (2012). Escalas de desenvolvimento infantil e o uso com bebês. Educar em Revista, 43, 81-100. doi:http://dx.doi.org/10.1590/S0104-40602012000100007

Rodrigues, O. M. P. R., \& Bolsoni-Silva, A. T. (2011). Efeitos da prematuridade sobre o desenvolvimento do lactante. Rev. Bras. Crescimento e Desenvolvimento Humano, 21(1), 111-121. Recuperado de http://pepsic.bvsalud.org/pdf/rbcdh/v21n1/11.pdf

Rodrigues, O. M. P. R., Tirelli, R. A., \& Silva, V. V. P. (2012). A influência de fatores de risco sobre o repertório comportamental de bebês. In: L. E. Melchiori; O. M. P. R. Rodrigues; A. C. B. Maia (Orgs.), Famílias e crianças: Reflexões teórico-práticas sobre os cuidados com as crianças (pp. 31-45). Curitiba: Juruá.

Sá, F. E., Nunes, N. P., Gondim, E. J. L., Almeida, A. K. F., Alencar, A. J. C., \& Cardoso, K. V. V. (2017). Intervenção parental melhora o desenvolvimento motor de lactentes de risco: Série de casos. Fisioterapia e Pesquisa, 24(1), 15-21. doi:http://dx.doi. org/10.1590/1809-2950/15828624012017

Saccani, R., Zanella, D. E., Notari, V. S, \& Valentini, N. C. (2017). Importância da correção da idade gestacional na avaliação motora de prematuros no primeiro ano de vida. Fisioterapia Brasil, 18(4), 409-416. Acessado de: http://docs.bvsalud.org/biblioref/2018/07/906511/ importancia-da-correcao-da-idade-gestacional-na-avaliacao-motor_9mFJzOz.pdf

Santos, J. C., \& Freitas, P. M. (2011). Planejamento familiar na perspectiva do desenvolvimento. Ciência \& Saúde Coletiva, 16(3), 1813-1820. Recuperado de: http://www.scielo.br/pdf/ csc/v16n3/17.pdf

Santos, N. L. A. C., Costa, M. C. O., Amaral, M. T. R., Vieira, G. O., Bacelar, E. B., \& Almeida, A. H. V. (2014). Gravidez na adolescência: Análise de fatores de risco para baixo peso, prematuridade e cesariana. Ciência e Saúde Coletiva, 19(3), 719-726. doi:http://dx.doi. org/10.1590/1413-81232014193.18352013

Santos, D. C. C., Tolocka, R. E., Carvalho, J., Heringer, L. R. C., Almeida, C. M., \& Miquelote, A. F. (2009). Desempenho motor grosso e sua associação com fatores neonatais, familiares e de exposição à creche em crianças até três anos de idade. Revista Brasileira de Fisioterapia, 13(2), 173-179. Recuperado de http://www.scielo.br/pdf/rbfis/v13n2/aop023_09.pdf

Sartori, N., Saccani, R., \& Valentini, N. C. (2010). Comparação do desenvolvimento motor de lactentes de mães adolescentes e adultas. Fisioterapia e Pesquisa, 17(4), 306-11. doi:http://dx.doi.org/10.1590/S1809-29502010000400004

Schiavo, R. A., Rodrigues, O. M. P. R., \& Perosa, G. B. (2018). Variáveis associadas à ansiedade gestacional em primigestas e multigestas. Temas em Psicologia, 26(4), 2091-2104. doi:http://dx.doi.org/10.9788/TP2018.4-14Pt

Seidl-de-Moura, M. L., Ribas, A. F. P., Seabra, K. C., Pessôa, L. F., Nogueira, S. E., Mendes, D. M. L. F., Rocha S. B., \& Vicente C. C. (2008). Interações mãe-bebê de um e cinco meses: Aspectos afetivos, complexidade e sistemas parentais predominantes. Psicologia: Reflexão e Crítica, 21(1), 66-73. doi:http://dx.doi.org/10.1590/S0102-79722008000100009

Severiano, A. A. O., Dantas, D. S., Oliveira, V. L. C., Lopes, J. M., Souza, D. S., \& Magalhães, A. G. (2017). Association between breastfeeding, obstetric factors and child development 
in northeast Brazil. J Hum Growth Dev. 27(2), 158-165. doi:http://dx.doi.org/10.7322/ jhgd.114483

Simas, F. B., Souza, L. V., \& Scorsolini-Comim, F. (2013). Significados da gravidez e da maternidade: Discurso de primíparas e multíparas. Revista Psicologia: Teoria e Prática, 15(1), 19-34. Recuperado de http://pepsic.bvsalud.org/pdf/ptp/v15n1/02.pdf

Silva, J. L. G. V., Soares, E. A., Caetano, E. A., Loyola, Y. C. S., Garcia, J. A. D., \& Mesquita G. (2011). O impacto da escolaridade materna e a renda per capita no desenvolvimento de crianças de zero a três anos. Revista Ciências em Saúde, 1(2), 62-67.

Silva, R. A. M., \& Magalhães, C. M. C. (2011). Crenças sobre práticas: um estudo sobre mães primíparas de contexto urbano e não-urbano. Revista Brasileira de Crescimento e Desenvolvimento Humano, 21(1), 39-50. doi:https://doi.org/10.7322/jhgd.19994

Tamis-Lemonda, C. S., \& Rodriguez, E. T. (2011). O papel dos pais na promoção da aprendizagem e no desenvolvimento da linguagem de crianças pequenas. In R. E. Tremblay, M. Boivin, R. D. E. V. Peters (Orgs.), Enciclopédia sobre o Desenvolvimento na Primeira Infância (pp. 1-9). Montreal, Quebec: Centre of Excellence for Early Childhood Development and Strategic Knowledge Cluster on Early Child Development. Recuperado de http://www.enciclopediacrianca.com/documents/Tamis-LeMonda-RodriguezPRTxp1-Parentais.pdf

Tella, P., Piccolo L. R., Rangel, M. L; Rohde, L. A., Polanczyk, G. V., Miguel, E. C., Grisi, S. J. F. E., Fleitlich-Bilyk, B., \& Ferraro, A. A. (2018). Socioeconomic diversities and infant development at 6 to 9 months in a poverty area of São Paulo, Brazil. Trends Psychiatry Psychother. 40(3), 232-240. Recuperado de http://www.scielo.br/pdf/trends/v40n3/22380019-trends-2237-6089-2017-0008.pdf

Word Health Organization. (2018). Preterm Birth. Genebra: WHO. Recuperado de http:// www.who.int/news-room/fact-sheets/detail/pretermBirth

Willrich, A., Azevedo, C. C. F., \& Fernandes, J. O. (2004). Desenvolvimento motor na infância: Influência dos fatores de risco e programas de intervenção. Cadernos de Saúde Pública, 20(3), 710-718. Recuperado de http://www.revistaneurociencias.com.br/edicoes/2009/ RN\%202009\%201/226\%20.pdf

Zago, J. T. C., Ferreira-Pinto, P. A., Ribeiro-Leite, H., Santos, J. N., \& Morais, R. L. S. (2017). Associação entre o desenvolvimento neuropsicomotor e fatores de risco biológicos e ambientais em crianças na primeira infância. Revista CEFAC., 19(3), 320-329. doi:http:// dx.doi.org/10.1590/1982-0216201719314416

Zajonz, R., Muller, A. B., \& Valentini, N. C. (2008). A influência de fatores ambientais no desempenho motor e social de crianças da periferia de porto alegre. Revista da Educação Física/UEM, 19(2), 159-171. Recuperado de:http://www.periodicos.uem.br/ojs/index. php/RevEducFis/article/view/3220/3496

Recebido em 18/06/2019

Última revisão: $31 / 07 / 2019$

Aceite final: 05/12/2019

\section{Sobre os autores:}

Rafaela de Almeida Schiavo: Pós-doutora em Psicologia do Desenvolvimento e Aprendizagem pela Universidade Estadual Paulista "Júlio de Mesquita Filho" (UNESP/Bauru). Docente dos cursos de Psicologia e Pedagogia da Universidade Paulista (UNIP/Bauru); diretora e fundado- 
ra do Instituto MaterOnline, Agudos, SP. E-mail: rafaela.schiavo@materonline.com.br, Orcid: https://orcid.org/0000-0001-7290-3610

Olga Maria Piazentin Rolim Rodrigues: Livre-docente em Psicologia pela Universidade Estadual Paulista "Júlio de Mesquita Filho" (UNESP/Bauru). Docente no curso de Psicologia e do curso de Pós-Graduação em Psicologia do Desenvolvimento e Aprendizagem da Universidade Estadual Paulista "Júlio de Mesquita Filho" (UNESP/Bauru). E-mail: olgarolim29@gmail.com, Orcid: https://orcid.org/0000-0002-5332-256X

Janaína Senhorini dos Santos: Mestranda em Fonoaudiologia pela Universidade Estadual Paulista "Júlio de Mesquita Filho" (Unesp). Graduada em Fisioterapia pela Universidade do Sagrado Coração. Fisioterapeuta na área Neurofuncional Infantil e Equoterapia, no Centro Especializado de Reabilitação SORRI-Bauru. E-mail: senhorinijanaina@yahoo.com.br, Orcid: https://orcid.org/0000-0003-1768-1837

Juliana Marinho Antonucci: Graduada em Fisioterapia. Fisioterapeuta no Centro Especializado de Reabilitação SORRI-Bauru. E-mail: superniptec@sorribauru.com.br, Orcid: https://orcid. org/0000-0002-3178-5299

Carolina Mormanno: Mestre em Psicologia do Desenvolvimento e Aprendizagem pela Universidade Estadual Paulista "Júlio de Mesquita Filho" (UNESP/Bauru). Graduada em Psicologia. E-mail: carolmormann0@gmail.com, Orcid: https://orcid.org/0000-0002-4178-2999

Verônica Aparecida Pereira: Doutora em Educação Especial pela Universidade Federal de São Carlos (UFSCar). Docente do curso de Psicologia da Universidade Federal da Grande Dourados (UFGD). E-mail: veronica.ufgd.tci@gmail.com, Orcid: https://orcid.org/0000-0002-9051-7550 
